\title{
Diastereoselective Decarboxylation of Cyclopentene Dicarboxylic Acid Derivatives
}

\author{
Jin Yong Lee, ${ }^{*}$, Jongseob Kim $^{\ddagger}{ }^{\ddagger}$ Ka Young Lee, ${ }^{\S}$ and Jae Nyoung Kim*, ${ }^{*}$ \\ Institute for Condensed Matter Theory and Department of Chemistry, Chonnam \\ National University, 300 Yongbong-Dong, Bukgu, Gwangju, 500-757, Korea, Electronic \\ Materials Laboratory, Samsung Advanced Institute of Technology, P.O.Box 111, Suwon \\ 440-600, Korea, Department of Chemistry and Institute of Basic Science, Chonnam \\ National University, 300 Yongbong-Dong, Bukgu, Gwangju, 500-757, Korea \\ E-mail: jinyong@chonnam.ac.kr
}

\section{Supporting Information}

\section{Contents}

Total energies and number of imaginary frequencies of $\mathbf{C O}_{2}, \mathbf{1}(\mathrm{R} 1, \mathrm{R} 2, \mathrm{~S} 1, \mathrm{~S} 2)$, TS1, TS2, R-Enol, 2 (trans, cis), 3 (trans, cis), 4 (trans, cis), 5 (trans, cis), 6 (trans, cis) (Table $\mathrm{S} 1$, in plain text)

Cartesian coordinates of $\mathbf{C O}_{2}, \mathbf{1}$ (R1, R2, S1, S2), TS1, TS2, R-Enol, $\mathbf{2}$ (trans, cis), $\mathbf{3}$ (trans, cis), 4 (trans, cis), 5 (trans, cis), 6 (trans, cis). (Table S2, in plain text). 
Table S1. B3LYP/6-31G* optimized energies for $\mathbf{C O}_{2}, \mathbf{1}$ (R1, R2, S1, S2), TS1, TS2, and R-Enol, B3LYP/6-311+G** single point energies (in Hartree) at B3LYP/6-31G* optimized geometries for $\mathbf{2}$ (trans, cis), $\mathbf{3}$ (trans, cis), $\mathbf{4}$ (trans, cis), 5 (trans, cis), 6 (trans, cis), and their number of imaginary frequency calculated at B3LYP/6-31G*.

\begin{tabular}{|c|c|c|c|}
\hline Molecule & Conformer & Energy & No imaginary freq \\
\hline $\mathrm{CO}_{2}$ & & -188.577570524 & 0 \\
\hline 1 & $\mathrm{R} 1$ & -803.489691466 & 0 \\
\hline 1 & $\mathrm{R} 2$ & -803.48791322 & 0 \\
\hline 1 & $\mathrm{~S} 1$ & -803.489691495 & 0 \\
\hline 1 & $\mathrm{~S} 2$ & -803.487913771 & 0 \\
\hline TS1 & & -803.452105368 & 1 \\
\hline $\mathrm{TS} 2$ & & -803.450082192 & 1 \\
\hline R-Enol & & -614.889938296 & 0 \\
\hline 2 & cis & -615.109704853 & 0 \\
\hline 2 & trans & -615.121639683 & 0 \\
\hline 3 & cis & -721.150730072 & 0 \\
\hline 3 & trans & -721.166968848 & 0 \\
\hline 4 & cis & -588.564211755 & 0 \\
\hline 4 & trans & -588.578511223 & 0 \\
\hline 5 & cis & -572.635849317 & 0 \\
\hline 5 & trans & -572.647158294 & 0 \\
\hline 6 & cis & -483.275082232 & 0 \\
\hline 6 & trans & -483.28440571 & 0 \\
\hline
\end{tabular}


Table S2. B3LYP/6-31G* optimized cartesian coordinates of $\mathbf{C O}_{2}, \mathbf{1}$ (R1, R2, S1, S2), TS1, TS2, R-Enol, 2 (trans, cis), 3 (trans, cis), 4 (trans, cis), 5 (trans, cis), 6 (trans, cis).

Cartesian coordinates of $\mathrm{CO}_{2}$

\begin{tabular}{cccccc}
\hline Number & Atomic number & & $\mathrm{x}$ & $\mathrm{y}$ & $\mathrm{z}$ \\
1 & 6 & 0 & 0.000000 & 0.000000 & 0.000000 \\
2 & 8 & 0 & 0.000000 & 0.000000 & 1.169599 \\
3 & 8 & 0 & 0.000000 & 0.000000 & -1.169599 \\
\hline
\end{tabular}

\section{Cartesian coordinates of $1(\mathrm{R} 1)$}

\begin{tabular}{|c|c|c|c|c|c|}
\hline Number & Atomic number & & $\mathrm{x}$ & $\mathrm{y}$ & $\mathrm{z}$ \\
\hline 1 & 6 & 0 & 1.376496 & -0.007110 & 0.114281 \\
\hline 2 & 6 & 0 & 0.296920 & -0.429228 & -1.017259 \\
\hline 3 & 6 & 0 & 0.689953 & -1.856420 & -1.325697 \\
\hline 4 & 6 & 0 & 1.846629 & -2.216174 & -0.772787 \\
\hline 5 & 6 & 0 & 2.478479 & -1.109621 & 0.029726 \\
\hline 6 & 1 & 0 & 0.086859 & -2.469133 & -1.989228 \\
\hline 7 & 1 & 0 & 2.333903 & -3.176639 & -0.912793 \\
\hline 8 & 1 & 0 & 2.811450 & -1.437403 & 1.019998 \\
\hline 9 & 1 & 0 & 3.347345 & -0.695689 & -0.493704 \\
\hline 10 & 1 & 0 & 0.524562 & 0.192065 & -1.894229 \\
\hline 11 & 6 & 0 & -1.162568 & -0.194227 & -0.666286 \\
\hline 12 & 6 & 0 & -1.939913 & -1.190181 & -0.058094 \\
\hline 13 & 6 & 0 & -3.279464 & -0.954315 & 0.256298 \\
\hline 14 & 6 & 0 & -3.860804 & 0.282429 & -0.029208 \\
\hline 15 & 6 & 0 & -3.094992 & 1.282351 & -0.632701 \\
\hline 16 & 6 & 0 & -1.757751 & 1.043591 & -0.949873 \\
\hline 17 & 1 & 0 & -1.491265 & -2.154442 & 0.164375 \\
\hline 18 & 1 & 0 & -3.870444 & -1.739806 & 0.720363 \\
\hline 19 & 1 & 0 & -4.904331 & 0.464669 & 0.212943 \\
\hline 20 & 1 & 0 & -3.539375 & 2.247180 & -0.861642 \\
\hline 21 & 1 & 0 & -1.166551 & 1.825549 & -1.421037 \\
\hline 22 & 6 & 0 & 1.935993 & 1.380926 & -0.304823 \\
\hline 23 & 6 & 0 & 0.687840 & 0.000211 & 1.471013 \\
\hline 24 & 8 & 0 & 2.811855 & 1.462660 & -1.135554 \\
\hline
\end{tabular}




\begin{tabular}{rrrrrr}
25 & 8 & 0 & 1.345733 & 2.470126 & 0.199282 \\
26 & 8 & 0 & 0.229357 & 0.987690 & 2.030122 \\
27 & 8 & 0 & 0.581079 & -1.215089 & 2.027793 \\
28 & 1 & 0 & 0.744536 & 2.200037 & 0.937428 \\
29 & 1 & 0 & 0.078207 & -1.092876 & 2.856839 \\
\hline
\end{tabular}

Cartesian coordinates of $1(\mathrm{R} 2)$

\begin{tabular}{|c|c|c|c|c|c|}
\hline Number & Atomic number & & $\mathrm{x}$ & $\mathrm{y}$ & $\mathrm{z}$ \\
\hline 1 & 6 & 0 & 1.362748 & -0.052247 & 0.153509 \\
\hline 2 & 6 & 0 & 0.275478 & -0.380188 & -1.008781 \\
\hline 3 & 6 & 0 & 0.650516 & -1.790644 & -1.403447 \\
\hline 4 & 6 & 0 & 1.795628 & -2.206638 & -0.867990 \\
\hline 5 & 6 & 0 & 2.437928 & -1.176733 & 0.021728 \\
\hline 6 & 1 & 0 & 0.028276 & -2.355052 & -2.091448 \\
\hline 7 & 1 & 0 & 2.256900 & -3.172137 & -1.053594 \\
\hline 8 & 1 & 0 & 2.682044 & -1.581947 & 1.007652 \\
\hline 9 & 1 & 0 & 3.363112 & -0.795674 & -0.423682 \\
\hline 10 & 1 & 0 & 0.496371 & 0.289024 & -1.853595 \\
\hline 11 & 6 & 0 & -1.182975 & -0.161217 & -0.649233 \\
\hline 12 & 6 & 0 & -1.915943 & -1.135666 & 0.043595 \\
\hline 13 & 6 & 0 & -3.259228 & -0.923104 & 0.352979 \\
\hline 14 & 6 & 0 & -3.890025 & 0.263987 & -0.025464 \\
\hline 15 & 6 & 0 & -3.169413 & 1.240345 & -0.715461 \\
\hline 16 & 6 & 0 & -1.826072 & 1.025825 & -1.025785 \\
\hline 17 & 1 & 0 & -1.425369 & -2.054075 & 0.351533 \\
\hline 18 & 1 & 0 & -3.813210 & -1.686346 & 0.892720 \\
\hline 19 & 1 & 0 & -4.937162 & 0.426580 & 0.215289 \\
\hline 20 & 1 & 0 & -3.652457 & 2.166168 & -1.016405 \\
\hline 21 & 1 & 0 & -1.269923 & 1.787689 & -1.568116 \\
\hline 22 & 6 & 0 & 1.865494 & 1.346878 & -0.144975 \\
\hline 23 & 6 & 0 & 0.677951 & -0.170416 & 1.548707 \\
\hline 24 & 8 & 0 & 2.888386 & 1.387347 & -1.015191 \\
\hline 25 & 8 & 0 & 1.361921 & 2.381561 & 0.274837 \\
\hline 26 & 8 & 0 & 0.112319 & 0.923970 & 2.063218 \\
\hline 27 & 8 & 0 & 0.630684 & -1.235308 & 2.120730 \\
\hline
\end{tabular}




\begin{tabular}{rrrrrr}
28 & 1 & 0 & 0.318104 & 1.701102 & 1.491272 \\
29 & 1 & 0 & 3.074315 & 2.333019 & -1.176673 \\
\hline
\end{tabular}

\section{Cartesian coordinates of $1(\mathrm{~S} 1)$}

\begin{tabular}{|c|c|c|c|c|c|}
\hline Number & Atomic number & & $\mathrm{x}$ & $\mathrm{y}$ & $\mathrm{z}$ \\
\hline 1 & 6 & 0 & -1.376699 & -0.007204 & 0.114318 \\
\hline 2 & 6 & 0 & -0.296980 & -0.427155 & -1.017856 \\
\hline 3 & 6 & 0 & -0.690024 & -1.853783 & -1.329036 \\
\hline 4 & 6 & 0 & -1.846563 & -2.214722 & -0.776595 \\
\hline 5 & 6 & 0 & -2.478458 & -1.109717 & 0.028018 \\
\hline 6 & 1 & 0 & -0.086712 & -2.465326 & -1.993442 \\
\hline 7 & 1 & 0 & -2.333518 & -3.175140 & -0.918018 \\
\hline 8 & 1 & 0 & -3.347578 & -0.694948 & -0.494305 \\
\hline 9 & 1 & 0 & -2.811024 & -1.439414 & 1.017729 \\
\hline 10 & 1 & 0 & -0.524352 & 0.195829 & -1.893704 \\
\hline 11 & 6 & 0 & 1.162572 & -0.193280 & -0.666438 \\
\hline 12 & 6 & 0 & 1.938493 & -1.189540 & -0.056912 \\
\hline 13 & 6 & 0 & 3.278134 & -0.954860 & 0.257954 \\
\hline 14 & 6 & 0 & 3.860996 & 0.280960 & -0.028471 \\
\hline 15 & 6 & 0 & 3.096621 & 1.281136 & -0.633351 \\
\hline 16 & 6 & 0 & 1.759250 & 1.043588 & -0.950928 \\
\hline 17 & 1 & 0 & 1.488686 & -2.153133 & 0.166131 \\
\hline 18 & 1 & 0 & 3.868012 & -1.740552 & 0.723083 \\
\hline 19 & 1 & 0 & 4.904599 & 0.462270 & 0.214059 \\
\hline 20 & 1 & 0 & 3.542220 & 2.245226 & -0.863046 \\
\hline 21 & 1 & 0 & 1.169132 & 1.825729 & -1.423133 \\
\hline 22 & 6 & 0 & -0.688043 & -0.001400 & 1.471048 \\
\hline 23 & 6 & 0 & -1.936211 & 1.381530 & -0.302617 \\
\hline 24 & 8 & 0 & -0.583461 & -1.216973 & 2.027715 \\
\hline 25 & 8 & 0 & -0.227643 & 0.985197 & 2.030122 \\
\hline 26 & 8 & 0 & -1.342599 & 2.469864 & 0.199639 \\
\hline 27 & 8 & 0 & -2.814614 & 1.464751 & -1.130486 \\
\hline 28 & 1 & 0 & -0.740867 & 2.199072 & 0.937020 \\
\hline 29 & 1 & 0 & -0.080586 & -1.095634 & 2.856893 \\
\hline
\end{tabular}




\section{Cartesian coordinates of $1(\mathrm{~S} 2)$}

\begin{tabular}{|c|c|c|c|c|c|}
\hline Number & Atomic number & & $\mathrm{x}$ & $\mathrm{y}$ & $\mathrm{z}$ \\
\hline 1 & 6 & 0 & -1.362950 & -0.052257 & 0.153194 \\
\hline 2 & 6 & 0 & -0.275461 & -0.379775 & -1.009117 \\
\hline 3 & 6 & 0 & -0.650511 & -1.790076 & -1.404334 \\
\hline 4 & 6 & 0 & -1.795556 & -2.206336 & -0.868928 \\
\hline 5 & 6 & 0 & -2.437976 & -1.176688 & 0.021016 \\
\hline 6 & 1 & 0 & -0.028247 & -2.354236 & -2.092512 \\
\hline 7 & 1 & 0 & -2.256730 & -3.171828 & -1.054808 \\
\hline 8 & 1 & 0 & -3.363092 & -0.795567 & -0.424444 \\
\hline 9 & 1 & 0 & -2.682230 & -1.582194 & 1.006784 \\
\hline 10 & 1 & 0 & -0.496187 & 0.289812 & -1.853688 \\
\hline 11 & 6 & 0 & 1.182975 & -0.161147 & -0.649329 \\
\hline 12 & 6 & 0 & 1.915562 & -1.135911 & 0.043460 \\
\hline 13 & 6 & 0 & 3.258832 & -0.923750 & 0.353164 \\
\hline 14 & 6 & 0 & 3.889997 & 0.263259 & -0.024927 \\
\hline 15 & 6 & 0 & 3.169756 & 1.239934 & -0.714865 \\
\hline 16 & 6 & 0 & 1.826418 & 1.025826 & -1.025500 \\
\hline 17 & 1 & 0 & 1.424702 & -2.054274 & 0.351111 \\
\hline 18 & 1 & 0 & 3.812515 & -1.687231 & 0.892873 \\
\hline 19 & 1 & 0 & 4.937127 & 0.425541 & 0.216059 \\
\hline 20 & 1 & 0 & 3.653089 & 2.165698 & -1.015524 \\
\hline 21 & 1 & 0 & 1.270523 & 1.787969 & -1.567680 \\
\hline 22 & 6 & 0 & -0.678239 & -0.170614 & 1.548461 \\
\hline 23 & 6 & 0 & -1.865184 & 1.347181 & -0.144700 \\
\hline 24 & 8 & 0 & -0.632832 & -1.235037 & 2.121477 \\
\hline 25 & 8 & 0 & -0.110556 & 0.923218 & 2.061957 \\
\hline 26 & 8 & 0 & -1.357930 & 2.381430 & 0.271727 \\
\hline 27 & 8 & 0 & -2.891976 & 1.388408 & -1.010259 \\
\hline 28 & 1 & 0 & -0.314015 & 1.700040 & 1.488862 \\
\hline 29 & 1 & 0 & -3.077074 & 2.334235 & -1.171818 \\
\hline
\end{tabular}




\section{Cartesian coordinates of TS1}

\begin{tabular}{|c|c|c|c|c|c|}
\hline Number & Atomic number & & $\mathrm{x}$ & $\mathrm{y}$ & $\mathrm{z}$ \\
\hline 1 & 6 & 0 & -1.334217 & 0.181906 & 0.136551 \\
\hline 2 & 6 & 0 & -0.229502 & 0.610997 & -0.967934 \\
\hline 3 & 6 & 0 & -0.541143 & 2.074143 & -1.188334 \\
\hline 4 & 6 & 0 & -1.677222 & 2.463906 & -0.613488 \\
\hline 5 & 6 & 0 & -2.372328 & 1.347628 & 0.120316 \\
\hline 6 & 1 & 0 & 0.097180 & 2.691424 & -1.813569 \\
\hline 7 & 1 & 0 & -2.109395 & 3.457009 & -0.694095 \\
\hline 8 & 1 & 0 & -2.689038 & 1.632530 & 1.128963 \\
\hline 9 & 1 & 0 & -3.261678 & 1.015871 & -0.426712 \\
\hline 10 & 1 & 0 & -0.489192 & 0.058037 & -1.881011 \\
\hline 11 & 6 & 0 & 1.213599 & 0.273764 & -0.633018 \\
\hline 12 & 6 & 0 & 2.043102 & 1.185144 & 0.035856 \\
\hline 13 & 6 & 0 & 3.366550 & 0.856108 & 0.334349 \\
\hline 14 & 6 & 0 & 3.879284 & -0.390780 & -0.028100 \\
\hline 15 & 6 & 0 & 3.061053 & -1.307035 & -0.692553 \\
\hline 16 & 6 & 0 & 1.740067 & -0.975096 & -0.993606 \\
\hline 17 & 1 & 0 & 1.647947 & 2.157092 & 0.318261 \\
\hline 18 & 1 & 0 & 3.998757 & 1.577083 & 0.846325 \\
\hline 19 & 1 & 0 & 4.910355 & -0.645440 & 0.201766 \\
\hline 20 & 1 & 0 & 3.451971 & -2.278874 & -0.981510 \\
\hline 21 & 1 & 0 & 1.107835 & -1.692089 & -1.512410 \\
\hline 22 & 6 & 0 & -0.740769 & -0.159638 & 1.440384 \\
\hline 23 & 8 & 0 & -0.679267 & 0.776024 & 2.379197 \\
\hline 24 & 8 & 0 & -0.318903 & -1.310692 & 1.694854 \\
\hline 25 & 1 & 0 & -0.285930 & 0.369224 & 3.177468 \\
\hline 26 & 1 & 0 & -0.816868 & -2.029080 & 0.843766 \\
\hline 27 & 6 & 0 & -2.087628 & -1.441253 & -0.501984 \\
\hline 28 & 8 & 0 & -1.486815 & -2.452489 & 0.018238 \\
\hline 29 & 8 & 0 & -2.950893 & -1.341786 & -1.329523 \\
\hline
\end{tabular}




\section{Cartesian coordinates of TS2}

\begin{tabular}{|c|c|c|c|c|c|}
\hline Number & Atomic number & & $\mathrm{x}$ & $\mathrm{y}$ & $\mathrm{z}$ \\
\hline 1 & 6 & 0 & -1.399857 & 0.091052 & 0.003161 \\
\hline 2 & 6 & 0 & -0.254474 & 0.592801 & -1.038346 \\
\hline 3 & 6 & 0 & -0.590096 & 2.057473 & -1.199672 \\
\hline 4 & 6 & 0 & -1.733958 & 2.412190 & -0.619293 \\
\hline 5 & 6 & 0 & -2.417727 & 1.272559 & 0.085260 \\
\hline 6 & 1 & 0 & 0.059702 & 2.710493 & -1.774612 \\
\hline 7 & 1 & 0 & -2.165114 & 3.408612 & -0.648577 \\
\hline 8 & 1 & 0 & -2.627036 & 1.511412 & 1.131823 \\
\hline 9 & 1 & 0 & -3.367383 & 1.020109 & -0.398076 \\
\hline 10 & 1 & 0 & -0.441556 & 0.082951 & -1.994793 \\
\hline 11 & 6 & 0 & 1.184260 & 0.288084 & -0.661188 \\
\hline 12 & 6 & 0 & 1.891066 & 1.103468 & 0.234516 \\
\hline 13 & 6 & 0 & 3.217713 & 0.816683 & 0.555525 \\
\hline 14 & 6 & 0 & 3.857718 & -0.286505 & -0.013520 \\
\hline 15 & 6 & 0 & 3.163057 & -1.104382 & -0.906284 \\
\hline 16 & 6 & 0 & 1.836337 & -0.815707 & -1.227547 \\
\hline 17 & 1 & 0 & 1.393106 & 1.954689 & 0.688668 \\
\hline 18 & 1 & 0 & 3.751558 & 1.456037 & 1.253365 \\
\hline 19 & 1 & 0 & 4.891987 & -0.506765 & 0.236853 \\
\hline 20 & 1 & 0 & 3.653478 & -1.963567 & -1.356110 \\
\hline 21 & 1 & 0 & 1.300246 & -1.453213 & -1.927658 \\
\hline 22 & 6 & 0 & -1.881782 & -1.243253 & -0.356523 \\
\hline 23 & 8 & 0 & -3.038921 & -1.369526 & -0.997166 \\
\hline 24 & 8 & 0 & -1.224488 & -2.272607 & -0.065984 \\
\hline 25 & 1 & 0 & -3.206604 & -2.325751 & -1.118011 \\
\hline 26 & 1 & 0 & -0.540975 & -1.931878 & 0.885391 \\
\hline 27 & 6 & 0 & -0.608574 & -0.175053 & 1.709930 \\
\hline 28 & 8 & 0 & -0.160820 & -1.373512 & 1.808568 \\
\hline 29 & 8 & 0 & -0.611209 & 0.763196 & 2.457784 \\
\hline
\end{tabular}




\section{Cartesian coordinates of R-Enol}

\begin{tabular}{|c|c|c|c|c|c|}
\hline Number & Atomic number & & $\mathrm{x}$ & $\mathrm{y}$ & $\mathrm{z}$ \\
\hline 1 & 6 & 0 & 1.586303 & 0.054171 & -0.134592 \\
\hline 2 & 6 & 0 & 0.467923 & -0.720469 & -0.836184 \\
\hline 3 & 6 & 0 & 0.897184 & -2.156661 & -0.585012 \\
\hline 4 & 6 & 0 & 2.060097 & -2.244613 & 0.065763 \\
\hline 5 & 6 & 0 & 2.654842 & -0.892895 & 0.383075 \\
\hline 6 & 1 & 0 & 0.296455 & -2.995113 & -0.925825 \\
\hline 7 & 1 & 0 & 2.554159 & -3.175728 & 0.329880 \\
\hline 8 & 1 & 0 & 2.857515 & -0.766435 & 1.457658 \\
\hline 9 & 1 & 0 & 3.626768 & -0.757120 & -0.118477 \\
\hline 10 & 1 & 0 & 0.468783 & -0.524635 & -1.922855 \\
\hline 11 & 6 & 0 & -0.943455 & -0.404758 & -0.339190 \\
\hline 12 & 6 & 0 & -1.315648 & -0.666160 & 0.989860 \\
\hline 13 & 6 & 0 & -2.598400 & -0.367146 & 1.442753 \\
\hline 14 & 6 & 0 & -3.537156 & 0.208347 & 0.578743 \\
\hline 15 & 6 & 0 & -3.181481 & 0.478441 & -0.741342 \\
\hline 16 & 6 & 0 & -1.893609 & 0.170081 & -1.195687 \\
\hline 17 & 1 & 0 & -0.587350 & -1.109857 & 1.663248 \\
\hline 18 & 1 & 0 & -2.869913 & -0.581565 & 2.473290 \\
\hline 19 & 1 & 0 & -4.536319 & 0.442974 & 0.935668 \\
\hline 20 & 1 & 0 & -3.902047 & 0.923157 & -1.422770 \\
\hline 21 & 1 & 0 & -1.625875 & 0.366338 & -2.232605 \\
\hline 22 & 6 & 0 & 1.617501 & 1.384724 & 0.014501 \\
\hline 23 & 8 & 0 & 2.670554 & 2.024768 & 0.585552 \\
\hline 24 & 8 & 0 & 0.657364 & 2.272632 & -0.377099 \\
\hline 25 & 1 & 0 & -0.184652 & 1.796773 & -0.500806 \\
\hline 26 & 1 & 0 & 2.394532 & 2.943635 & 0.739846 \\
\hline
\end{tabular}


Cartesian coordinates of 2 (cis)

\begin{tabular}{|c|c|c|c|c|c|}
\hline Number & Atomic number & & $\mathrm{x}$ & $\mathrm{y}$ & $\mathrm{z}$ \\
\hline 1 & 6 & 0 & 1.606899 & 0.197868 & -0.784373 \\
\hline 2 & 6 & 0 & 0.575039 & -0.993010 & -0.579656 \\
\hline 3 & 6 & 0 & 1.294667 & -1.870546 & 0.426705 \\
\hline 4 & 6 & 0 & 2.581368 & -1.545932 & 0.557585 \\
\hline 5 & 6 & 0 & 2.967763 & -0.375771 & -0.311926 \\
\hline 6 & 1 & 0 & 0.792381 & -2.696010 & 0.922623 \\
\hline 7 & 1 & 0 & 3.297310 & -2.069892 & 1.184270 \\
\hline 8 & 1 & 0 & 3.544247 & 0.383426 & 0.224364 \\
\hline 9 & 1 & 0 & 3.573193 & -0.709526 & -1.167106 \\
\hline 10 & 1 & 0 & 0.525964 & -1.532442 & -1.538215 \\
\hline 11 & 6 & 0 & -0.853687 & -0.620889 & -0.220688 \\
\hline 12 & 6 & 0 & -1.228229 & -0.348429 & 1.104008 \\
\hline 13 & 6 & 0 & -2.540001 & 0.007757 & 1.414641 \\
\hline 14 & 6 & 0 & -3.502818 & 0.102897 & 0.406381 \\
\hline 15 & 6 & 0 & -3.145589 & -0.165668 & -0.915399 \\
\hline 16 & 6 & 0 & -1.831689 & -0.529705 & -1.223579 \\
\hline 17 & 1 & 0 & -0.481922 & -0.411022 & 1.891620 \\
\hline 18 & 1 & 0 & -2.810672 & 0.215574 & 2.446271 \\
\hline 19 & 1 & 0 & -4.523989 & 0.382160 & 0.650525 \\
\hline 20 & 1 & 0 & -3.888249 & -0.102882 & -1.706331 \\
\hline 21 & 1 & 0 & -1.564095 & -0.760624 & -2.253356 \\
\hline 22 & 6 & 0 & 1.276472 & 1.497893 & -0.029037 \\
\hline 23 & 1 & 0 & 1.629894 & 0.470686 & -1.846952 \\
\hline 24 & 8 & 0 & 0.163640 & 2.155454 & -0.439069 \\
\hline 25 & 8 & 0 & 1.972836 & 1.983739 & 0.829280 \\
\hline 26 & 1 & 0 & -0.387042 & 1.578208 & -0.997377 \\
\hline
\end{tabular}


Cartesian coordinates of 2 (trans)

\begin{tabular}{|c|c|c|c|c|c|}
\hline Number & Atomic number & & $\mathrm{x}$ & $\mathrm{y}$ & $\mathrm{z}$ \\
\hline 1 & 6 & 0 & -1.401880 & 0.075809 & 0.368577 \\
\hline 2 & 6 & 0 & -0.361035 & 0.568647 & -0.682620 \\
\hline 3 & 6 & 0 & -0.701067 & 2.042044 & -0.796708 \\
\hline 4 & 6 & 0 & -1.875667 & 2.344090 & -0.238968 \\
\hline 5 & 6 & 0 & -2.550296 & 1.134930 & 0.364232 \\
\hline 6 & 1 & 0 & -0.058304 & 2.738914 & -1.327096 \\
\hline 7 & 1 & 0 & -2.343775 & 3.324523 & -0.253746 \\
\hline 8 & 1 & 0 & -2.951845 & 1.305052 & 1.369561 \\
\hline 9 & 1 & 0 & -3.397058 & 0.812592 & -0.260259 \\
\hline 10 & 1 & 0 & -0.579966 & 0.072436 & -1.638230 \\
\hline 11 & 6 & 0 & 1.075415 & 0.240922 & -0.310847 \\
\hline 12 & 6 & 0 & 1.803369 & 1.054393 & 0.568972 \\
\hline 13 & 6 & 0 & 3.111952 & 0.727016 & 0.927648 \\
\hline 14 & 6 & 0 & 3.713241 & -0.423748 & 0.413387 \\
\hline 15 & 6 & 0 & 2.996253 & -1.243456 & -0.460635 \\
\hline 16 & 6 & 0 & 1.688743 & -0.912095 & -0.819858 \\
\hline 17 & 1 & 0 & 1.340725 & 1.952756 & 0.971172 \\
\hline 18 & 1 & 0 & 3.662686 & 1.372361 & 1.607441 \\
\hline 19 & 1 & 0 & 4.732872 & -0.678421 & 0.690382 \\
\hline 20 & 1 & 0 & 3.455704 & -2.141298 & -0.866197 \\
\hline 21 & 1 & 0 & 1.129183 & -1.555102 & -1.494593 \\
\hline 22 & 6 & 0 & -1.900620 & -1.328855 & 0.114215 \\
\hline 23 & 1 & 0 & -0.938314 & 0.087549 & 1.361240 \\
\hline 24 & 8 & 0 & -1.745176 & -1.979243 & -0.897783 \\
\hline 25 & 8 & 0 & -2.597450 & -1.804837 & 1.177013 \\
\hline 26 & 1 & 0 & -2.901351 & -2.696909 & 0.922116 \\
\hline
\end{tabular}




\begin{tabular}{cccccc}
\multicolumn{6}{c}{ Cartesian coordinates of 3 (cis) } \\
\hline Number & AN & & $\mathrm{x}$ & $\mathrm{y}$ & $\mathrm{z}$ \\
1 & 6 & 0 & -0.989593 & -0.036691 & -0.807709 \\
2 & 6 & 0 & 0.304201 & 0.815280 & -0.536173 \\
3 & 6 & 0 & -0.195609 & 1.943689 & 0.343208 \\
4 & 6 & 0 & -1.524265 & 2.035391 & 0.299258 \\
5 & 6 & 0 & -2.143839 & 0.976521 & -0.577717 \\
6 & 1 & 0 & 0.481464 & 2.603892 & 0.874442 \\
7 & 1 & 0 & -2.112929 & 2.794841 & 0.804541 \\
8 & 1 & 0 & -3.002157 & 0.487163 & -0.110154 \\
9 & 1 & 0 & -2.482365 & 1.408166 & -1.530554 \\
10 & 1 & 0 & 0.660174 & 1.221225 & -1.494954 \\
11 & 6 & 0 & -1.193435 & -1.279383 & 0.089677 \\
12 & 1 & 0 & -0.973076 & -0.401123 & -1.841310 \\
13 & 8 & 0 & -0.397530 & -2.341892 & -0.176990 \\
14 & 8 & 0 & -2.051955 & -1.367594 & 0.930989 \\
15 & 1 & 0 & 0.321858 & -2.097907 & -0.783674 \\
16 & 6 & 0 & 1.516490 & 0.100282 & 0.027056 \\
17 & 9 & 0 & 2.557364 & 0.947865 & 0.142280 \\
18 & 9 & 0 & 1.923406 & -0.903863 & -0.815894 \\
19 & 9 & 0 & 1.303587 & -0.451880 & 1.231846 \\
\hline
\end{tabular}




\section{Cartesian coordinates of 3 (trans)}

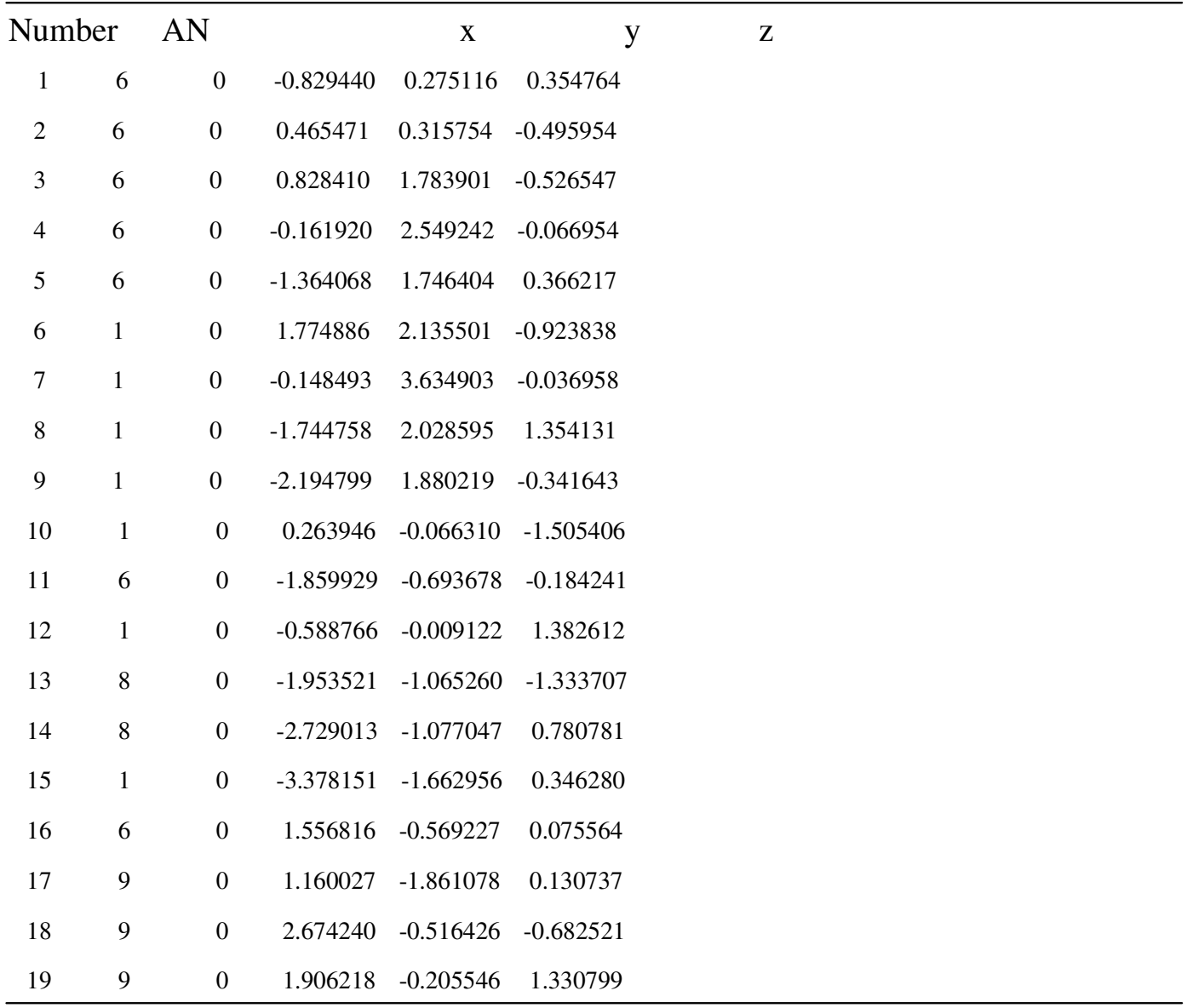




\section{Cartesian coordinates of 4 (cis)}

\begin{tabular}{cllrrrl}
\hline Number & AN & & \multicolumn{2}{r}{$\mathrm{y}$} & $\mathrm{Z}$ \\
1 & 6 & 0 & -0.721227 & 0.232361 & 0.787571 \\
2 & 6 & 0 & 0.336308 & -0.896765 & 0.537216 \\
3 & 6 & 0 & -0.370063 & -1.884375 & -0.347530 \\
4 & 6 & 0 & -1.689055 & -1.693927 & -0.280901 \\
5 & 6 & 0 & -2.059991 & -0.532847 & 0.608928 \\
6 & 1 & 0 & 0.154333 & -2.667282 & -0.880381 \\
7 & 1 & 0 & -2.431581 & -2.313879 & -0.773403 \\
8 & 1 & 0 & -2.822189 & 0.115175 & 0.170258 \\
9 & 1 & 0 & -2.434743 & -0.890362 & 1.578351 \\
10 & 1 & 0 & 0.627974 & -1.337435 & 1.499892 \\
11 & 6 & 0 & -0.673916 & 1.437704 & -0.187862 \\
12 & 1 & 0 & -0.601708 & 0.634773 & 1.798682 \\
13 & 8 & 0 & 0.376402 & 2.268881 & -0.055378 \\
14 & 8 & 0 & -1.552018 & 1.678707 & -0.979267 \\
15 & 1 & 0 & 1.058459 & 1.885260 & 0.532109 \\
16 & 7 & 0 & 1.680421 & -0.422196 & 0.009446 \\
17 & 8 & 0 & 2.259621 & 0.433299 & 0.693960 \\
18 & 8 & 0 & 2.135267 & -0.936359 & -0.996335 \\
\hline & & & & &
\end{tabular}




\section{Cartesian coordinates of 4 (trans)}

\begin{tabular}{cccrcr}
\hline Number & AN & & $\mathrm{x}$ & $\mathrm{y}$ & $\mathrm{z}$ \\
1 & 6 & 0 & 0.607180 & 0.176315 & -0.376327 \\
2 & 6 & 0 & -0.690493 & 0.101449 & 0.443265 \\
3 & 6 & 0 & -1.193092 & 1.518353 & 0.517390 \\
4 & 6 & 0 & -0.307858 & 2.374198 & 0.003171 \\
5 & 6 & 0 & 0.941017 & 1.700133 & -0.503571 \\
6 & 1 & 0 & -2.148222 & 1.760414 & 0.967611 \\
7 & 1 & 0 & -0.435661 & 3.452462 & -0.026982 \\
8 & 1 & 0 & 1.189591 & 1.978070 & -1.533996 \\
9 & 1 & 0 & 1.811090 & 1.975620 & 0.107334 \\
10 & 1 & 0 & -0.532022 & -0.354472 & 1.426634 \\
11 & 6 & 0 & 1.731075 & -0.629043 & 0.242799 \\
12 & 1 & 0 & 0.419650 & -0.240535 & -1.369570 \\
13 & 8 & 0 & 1.692484 & -1.211735 & 1.304447 \\
14 & 8 & 0 & 2.830724 & -0.613986 & -0.544990 \\
15 & 1 & 0 & 3.508721 & -1.141896 & -0.081459 \\
16 & 7 & 0 & -1.747676 & -0.819010 & -0.182925 \\
17 & 8 & 0 & -1.435795 & -1.529941 & -1.131328 \\
18 & 8 & & -2.850712 & -0.787465 & 0.350689 \\
& & 0 & & &
\end{tabular}




\section{Cartesian coordinates of 5 (cis)}

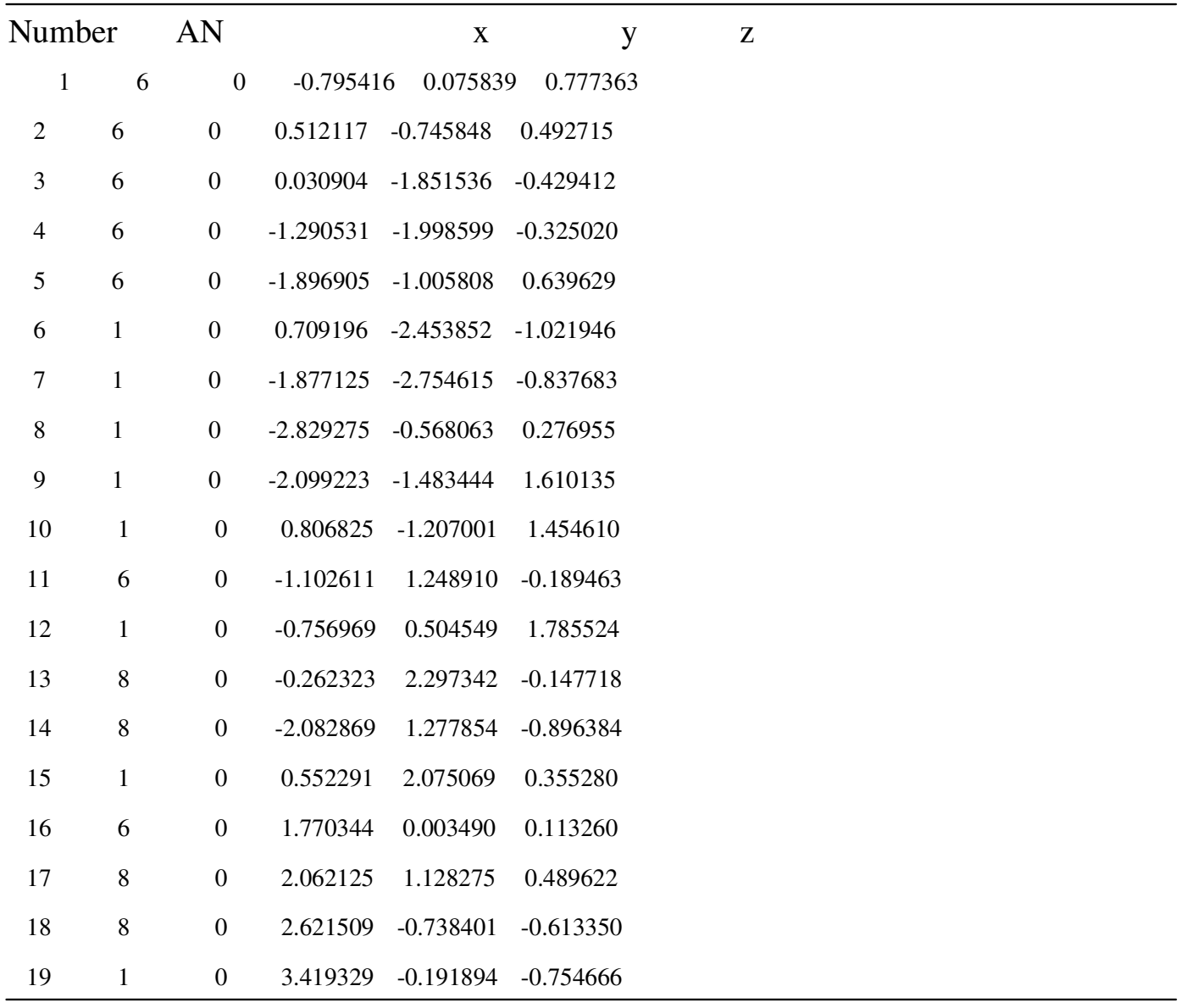




\section{Cartesian coordinates of 5 (trans)}

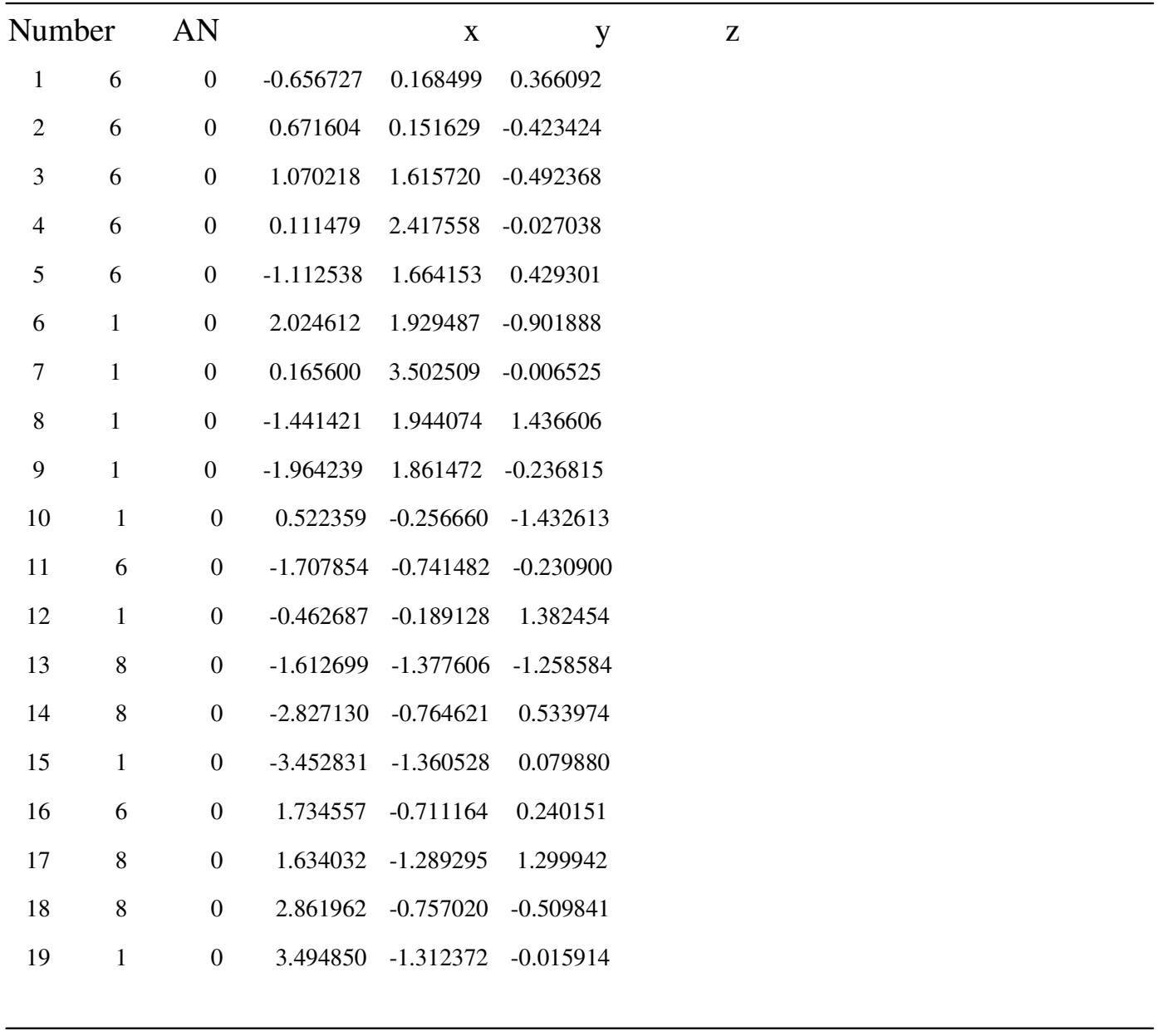




\begin{tabular}{ccccccc}
\multicolumn{8}{c}{ Cartesian coordinates of 6 (cis) } & \multicolumn{1}{c}{ x } & y & Z \\
\hline Number & \multicolumn{7}{c}{ Atomic number } & & \\
1 & 6 & 0 & -0.081745 & -0.285339 & 0.753462 & \\
2 & 6 & 0 & 0.914666 & 0.870269 & 0.457965 & \\
3 & 6 & 0 & 1.871524 & 0.286092 & -0.538668 & \\
4 & 6 & 0 & 1.796398 & -1.047062 & -0.533730 & \\
5 & 6 & 0 & 0.746399 & -1.557259 & 0.425192 & \\
6 & 1 & 0 & 2.566255 & 0.901550 & -1.100221 & \\
7 & 1 & 0 & 2.430281 & -1.710736 & -1.114238 & \\
8 & 1 & 0 & 0.106971 & -2.339284 & 0.007425 & \\
9 & 1 & 0 & 1.215376 & -1.966119 & 1.330927 & \\
10 & 1 & 0 & 1.420227 & 1.197261 & 1.374901 & \\
11 & 6 & 0 & -1.413555 & -0.304587 & -0.041950 & \\
12 & 1 & 0 & -0.387914 & -0.262569 & 1.806596 & \\
13 & 8 & 0 & -2.003400 & 0.877979 & -0.286224 & \\
14 & 8 & 0 & -1.955027 & -1.340961 & -0.343715 & \\
15 & 1 & 0 & -1.361942 & 1.598174 & -0.129313 & \\
16 & 9 & 0 & 0.297337 & 2.056989 & -0.030021 & \\
\hline
\end{tabular}




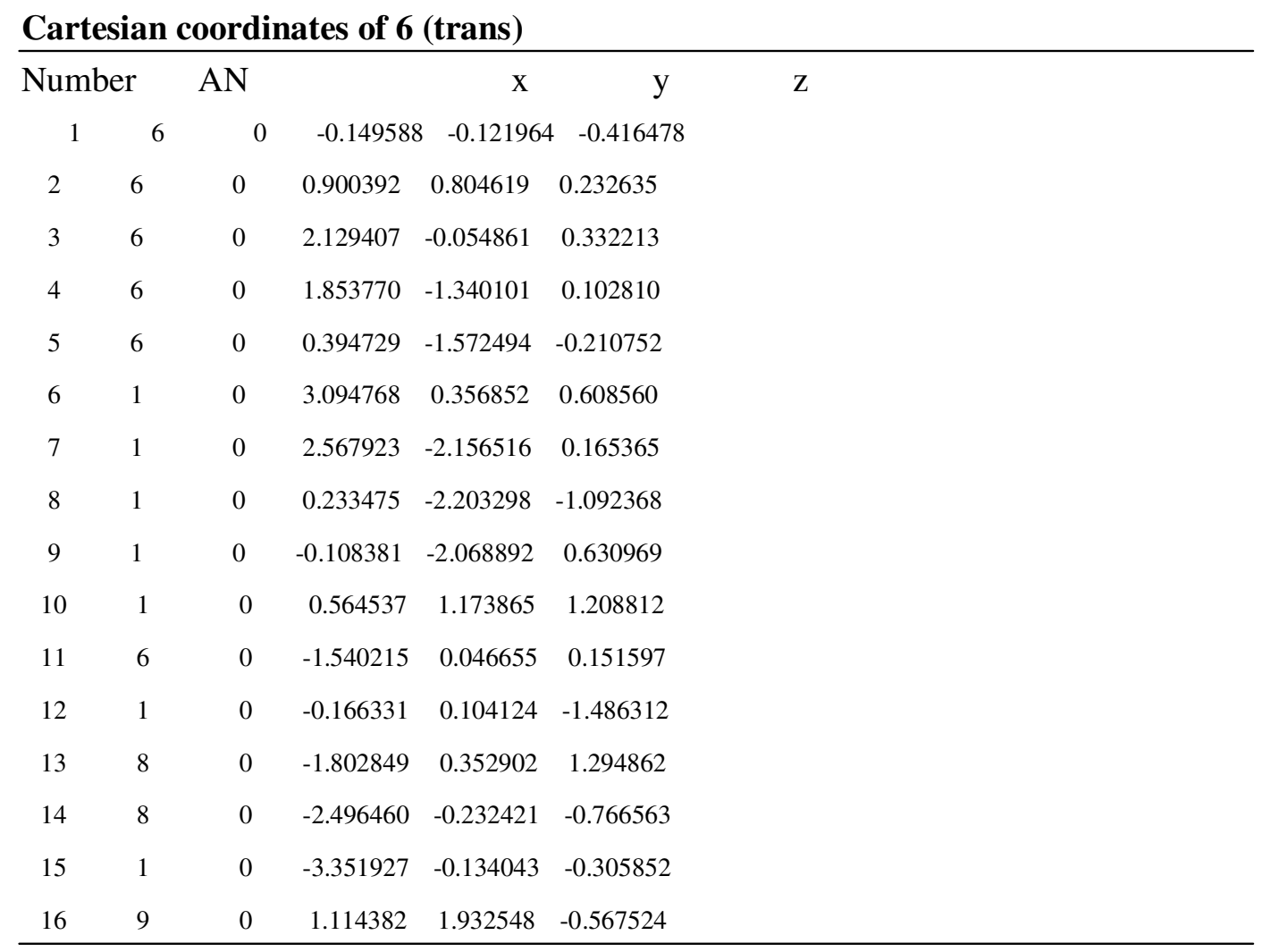

